\title{
Surface Binding of Lysine to Nanoporous PLGA Microparticles
}

\author{
Mohammad G Abdallah ${ }^{1,2 *}$ and Samir M Iqbal ${ }^{3}$ \\ ${ }^{1}$ Department of Electrical Engineering, the University of Texas at Arlington, Arlington, TX 76019, USA \\ ${ }^{2}$ Nanotechnology Research Center, the University of Texas at Arlington, Arlington, TX 76019, USA \\ ${ }^{3}$ Nano-bio Lab, Department of Electrical Engineering, and School of Medicine, University of Texas Rio Grande Valley, Edinburg, \\ TX 78539, USA \\ *Corresponding author: Mohammad G. Abdallah, Department of Electrical Engineering, the University of Texas at Arlington, \\ Arlington, USA
}

\section{ARTICLE INFO}

Received: 慧 April 14, 2020

Published: 慧 April 23, 2020

Citation: Mohammad G A, Samir M I. Surface Binding of Lysine to Nanoporous PLGA Microparticles. Biomed J Sci \& Tech Res 27(2)-2020. BJSTR. MS.ID.004465.

Keywords: Cell Targeting; PLGA; Ion Channels; Nanoporous; Target Delivery; Salt-Leaching.

\section{ABSTRACT}

Polylactic-co-glycolic acid (PLGA) microparticles have attracted scientists and engineers from various research fields. PLGA microparticles have been extensively used for drug delivery since the synthesized polymer is FDA approved polymer. Here, we present a method to control the formation of PLGA microparticles. prose and the surface activation of microparticles. A series of nanoporous PLGA microparticles were synthesized with different surface porosity. The porosity agent concentration influenced the surface activation. Thus, nanoporous PLGA microparticle can be introduced as a new focused drug delivery platform. The porosity of the activated microparticles showed a direct relationship with the covalent attachment of Lysine primary amine molecule. The nanoporous PLGA microparticle synthesis is a fast and low-cost method. Furthermore, nanoporous PLGA microparticles are suitable for applications in targeting one or multiple extracellular ligands. In specific, cancer cell ion channels exist in the cell 's extracellular space and can be targeted without entering the target cells.

\section{Introduction}

PLGA has been widely used for drug delivery and shown significant improvement in therapeutic areas. PLGA blocks are built from lactic and glycolic acid monomers that can be absorbed by the cell. Therefore, PLGA is a non-immunogenic, non-toxic, and stable polymer for the development of drug delivery. Furthermore, hydrophilic and hydrophobic drug molecules, DNA, and proteins can be encapsulated [1]. PLGA has an excellent biocompatibility profile [2,3]; therefore, the encapsulated molecules achieved target release profile through degradation and erosion of the polymer matrix $[4,5]$.

Nanotechnology offers unique and fascinating approaches in the area of nanomedicine and healthcare application [6,7]. Nanoporous microparticles have been used for bioseparation, drug release, and tissue engineering. In bioseparation, highly nanoporous microspheres have been extensively commercialized in chromatography [8-10]. In drug release, nanoporous microspheres can be designed to be multifunctional carriers for efficiently loading drugs [11,12]. The porous nanoparticles showed higher drug loading and releasing over a period of 30 days from nonporous nanoparticles [13]. In porous PLGA microparticles, autocatalytic effects play an essential role in controlling the drug release. The presence of the nanopores increases the drug molecules' mobility and altered the drug release mechanisms [14]. In tissue engineering and regenerative medicine, nanoporous microspheres are one of the best candidates for regenerative repair, and tissue engineering $[15,16]$. These nanoporous microparticles show significant advantages in many practical applications.

The primary aims of this study:

(i) Surface activate nanoporous PLGA microparticles (Figure 1), specifically, the crosslinking of bis (sulfosuccinimidyl) suberate (BS3) with primary amines to form amide bonds.

(ii) Characterize the effect of salt concentration on the formation of nanoporous and microparticles porosity.

(iii) study the effects of the porosity on the covalent amide bond formed between primary amine ligand and BS3 molecules. 
We introduce a method that can be used to target extracellular ligand, such as cell ion channels. The channel blockers may crosslink to nanoporous PLGA microparticles and act like a cork in a bottle, preventing the flow of ions in targeted cells until it diffuses off gradually. A targeted cell approach blocking ion channels activity can provide controlled, and a better-tolerated treatment for patients.

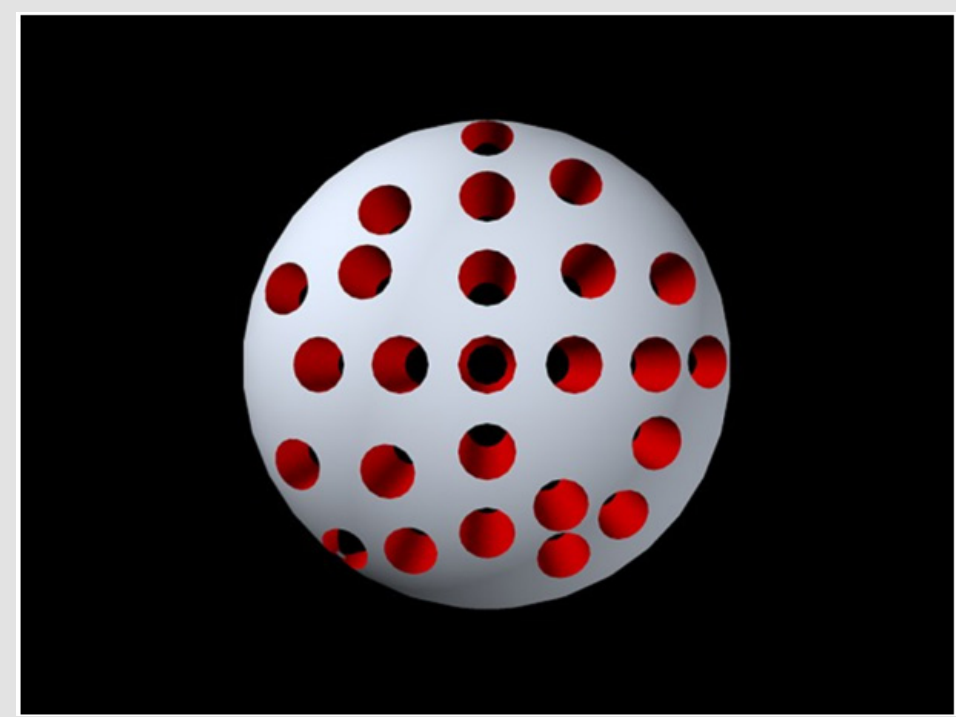

Figure 1: A 3D sketch of nanoporous PLGA microparticles.

\section{Materials and Methods}

\section{Materials}

All analytical grade reagents used in this work are purchased from Sigma-Aldrich (St. Louis, MO) unless noted otherwise. Poly (D, L-lactide-co-glycolide) 75:25 molar ratio with a carbocyclic group (uncapped) was obtained from Resomer® (Darmstadt, Germany). 1, 2-Dichloroethane (ACS reagent, $\geq 99 \%$ ) polyvinylpyrrolidone (PVP-k90), Lysine, and sodium bicarbonate (NaHCO3) were purchased from Sigma-Aldrich (St. Louis, Missouri, USA). Micro BCA protein assay kit and bis (sulfosuccinimidyl) suberate (BS3) were purchased from Thermo-Scientific, Rockford, Illinois, USA.

\section{Synthesis of Nanoporous PLGA Microparticles}

PLGA microparticles were synthesized using a double emulsion method [17]. First, 3\% (w/v) of the PLGA solution was prepared by dissolving PLGA in dichloroethane. Then, $0.2 \mathrm{~mL}$ of deionized (DI) water was added to the PLGA solution and vortexed for $30 \mathrm{sec}$ to create a water-in-oil phase. A solution of $3 \%, 0.5 \%$, and $0.1 \%(\mathrm{w} / \mathrm{v})$ of sodium bicarbonate in DI water were prepared. Then, the sodium bicarbonate solution was added to the water-in-oil phase sample to form nanoporous microparticles. Then, $5 \%$ of PVP-k90 solution in DI water was prepared and two mg of BS3 dissolved in DI water $(100 \mu \mathrm{L})$ was added to PVP-K90 solution and vortexed for one minute to ensure well mixing. The step of adding $\mathrm{NaHCO}_{3}$ solution was skipped for the preparation of nonporous PLGA microparticles, as shown by the schematic flowchart (Scheme 1). It was then added dropwise to the stirring beaker of PVP-k90 solution. Table 1 shows the prepared samples. Next, the solution vials were kept on stir plate overnight in a chemical hood to allow dichloroethane to evaporate. Once the dichloroethane evaporated entirely, the solution was transferred into a $15 \mathrm{ml}$ tube and centrifuged at $4000 \mathrm{rpm}$ for 15 $\mathrm{min}$. The pellets were collected and suspended in phosphate buffer saline (PBS), then freeze-dried at $-80^{\circ} \mathrm{C}$.

The freeze-dried microparticles were taken in a plastic tube, washed with DI water, and centrifuged at 4,000 rpm for $15 \mathrm{~min}$ three times to form nanoporous PLGA microparticles and remove the trapped salt. Iterative washing in DI water after centrifugation is known to remove the water-soluble salts completely $[13,18,19]$. The pellets were suspended in $5 \mathrm{~mL}$ PBS. ZetaPALS DLS detector (Brookhaven Instruments, Holtsville, NY, USA) was used to measure the size and polydispersity of microparticles

Table 1: Percent composition $(\mathrm{w} / \mathrm{v})$ of synthesized PLGA microparticles.

\begin{tabular}{|c|c|c|c|}
\hline \multirow{2}{*}{ Sample No. } & \multicolumn{3}{|c|}{ Components \% (w/v) } \\
\cline { 2 - 4 } & PLGA & PVP-k90 & NaHCO $_{3}$ \\
\hline A & 3 & 5 & 3 \\
\hline B & 3 & 5 & 0.5 \\
\hline C & 3 & 5 & 0.1 \\
\hline D & 3 & 5 & n/a \\
\hline
\end{tabular}




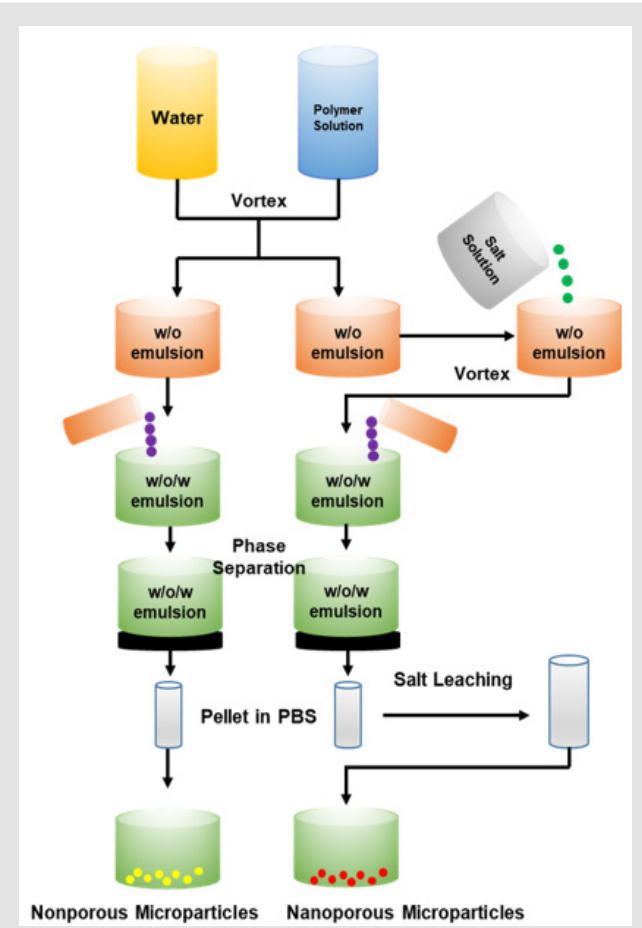

Scheme 1: Schematic flowchart for the procedure to synthesize nanoporous and nonporous microparticles. PLGA microparticles were synthesized using double emulsion method. DI water was added to PLGA solution and vortexed, then $\mathrm{NaHCO}_{3}$ was prepared and added to water-in-oil phase samples. The sample was added dropwise to solution of PVP-K90 with BS3. The sample was kept overnight on stir plate to complete the phase separation. Finally, the sample was washed with DI water to perform salt leaching step.

\section{Preparation of Nanoporous PLGA Microparticles for Scanning Electron Microscope (SEM) Imaging}

$10 \mathrm{mg}$ freeze-dried microparticles were suspended in $1.5 \mathrm{~mL}$ DI water; the sample was diluted for imaging in SEM. DI water was used instead of PBS to avoid crystallization and imaging artifacts stemming from the salts. The sample was sputter-coated with $50 \AA$ thick gold to prevent the surface from getting charged during SEM imaging. The thin layer of gold coating made sure that the glass coverslip surface was conductive enough to image with SEM.

\section{Covalent Attachment of Lysine to Nanoporous PLGA Microparticles}

The surface activated nanoporous PLGA microparticles were suspended in PBS for covalent attachment to Lysine. The microparticles underwent the process of conjugation, followed by PBS washing. For each sample in Table 1, $10 \mathrm{mg}$ was suspended in $5 \mathrm{~mL}$ PBS, then $30 \mathrm{mg}$ of Lysine was added to each sample and incubated for $30 \mathrm{~min}$. The excess of Lysine was removed by centrifugation followed by PBS wash $\left(\mathrm{x}_{2}\right)$ to obtain Lysine conjugated to nanoporous microparticles surface.

\section{Result}

\section{Nanoporous PLGA Microparticle Morphology}

The shape of nanoporous PLGA microparticles is a critical feature in the success of targeting drug delivery [20]. Also, the shape of microparticle influences the surface area available for surface activation. Here, the nanoporous microparticles were prepared using the double emulsion method with $\mathrm{NaHCO} 3$ as the pores foaming agent. The morphology of synthesized nanoporous PLGA microparticles was found to be spherical from SEM micrographs (Figure 2). PLGA microparticles with large surface-to-volume ratios are preferred for targeting since sizable surfaces allow for the attaching of many ligands. 


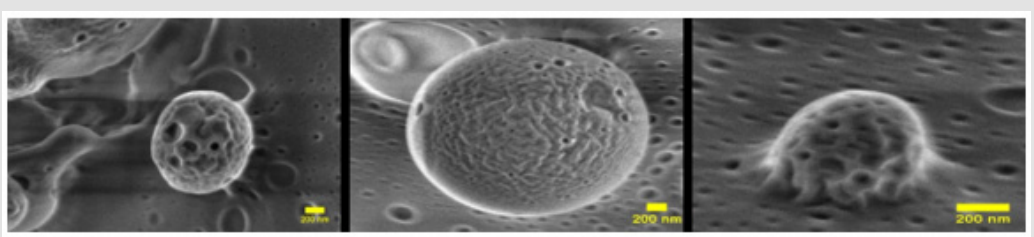

(a)

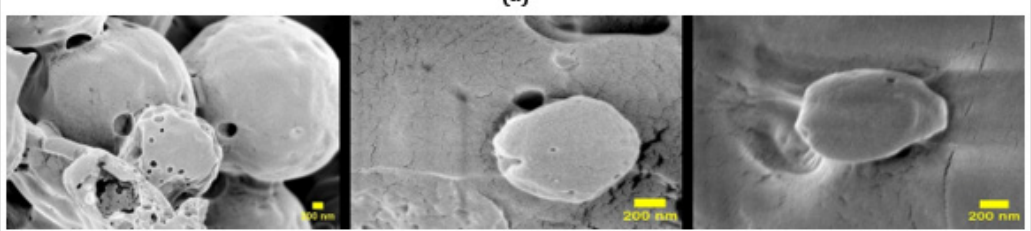

(b)

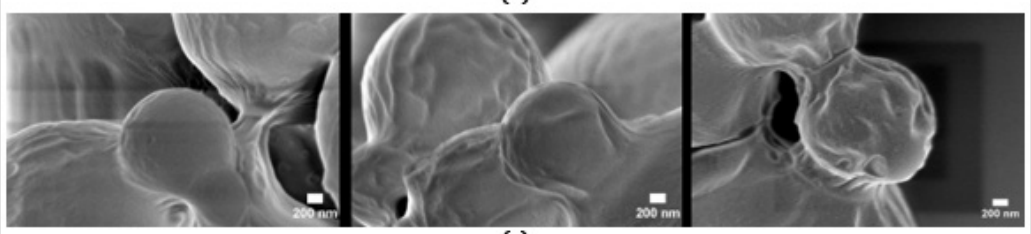

(c)

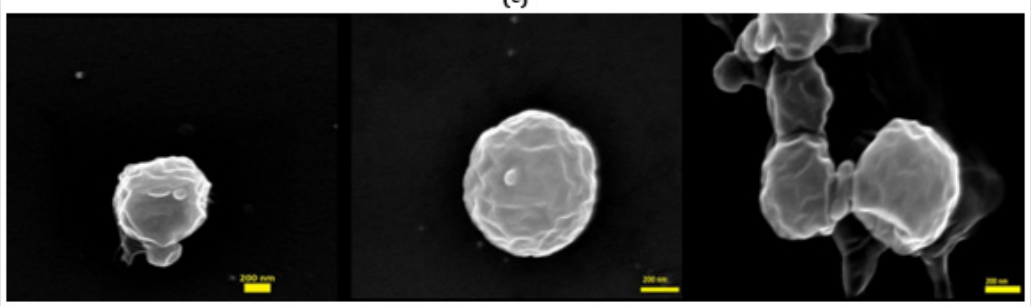

(d)

Figure 2: SEM images of 3\% PLGA microparticles prepared using double emulsion method with different salt concentration. (a) $3 \% \mathrm{NaHCO}_{3}$ (b) $0.5 \% \mathrm{NaHCO}_{3}$ (c) $0.1 \% \mathrm{NaHCO}_{3}$ (d) nonporous PLGA microparticles (Scale bar = $200 \mathrm{~nm}$ )

\section{Nanoporous PLGA Microparticle Size}

The size distribution of the microparticle samples was measured using a ZetaPALS DLS detector. The mean diameter of the nonporous PLGA microparticles was $0.60 \pm 0.30 \mu \mathrm{m}$ (polydispersity = 0.37). The diameter of nanoporous PLGA microparticles prepared
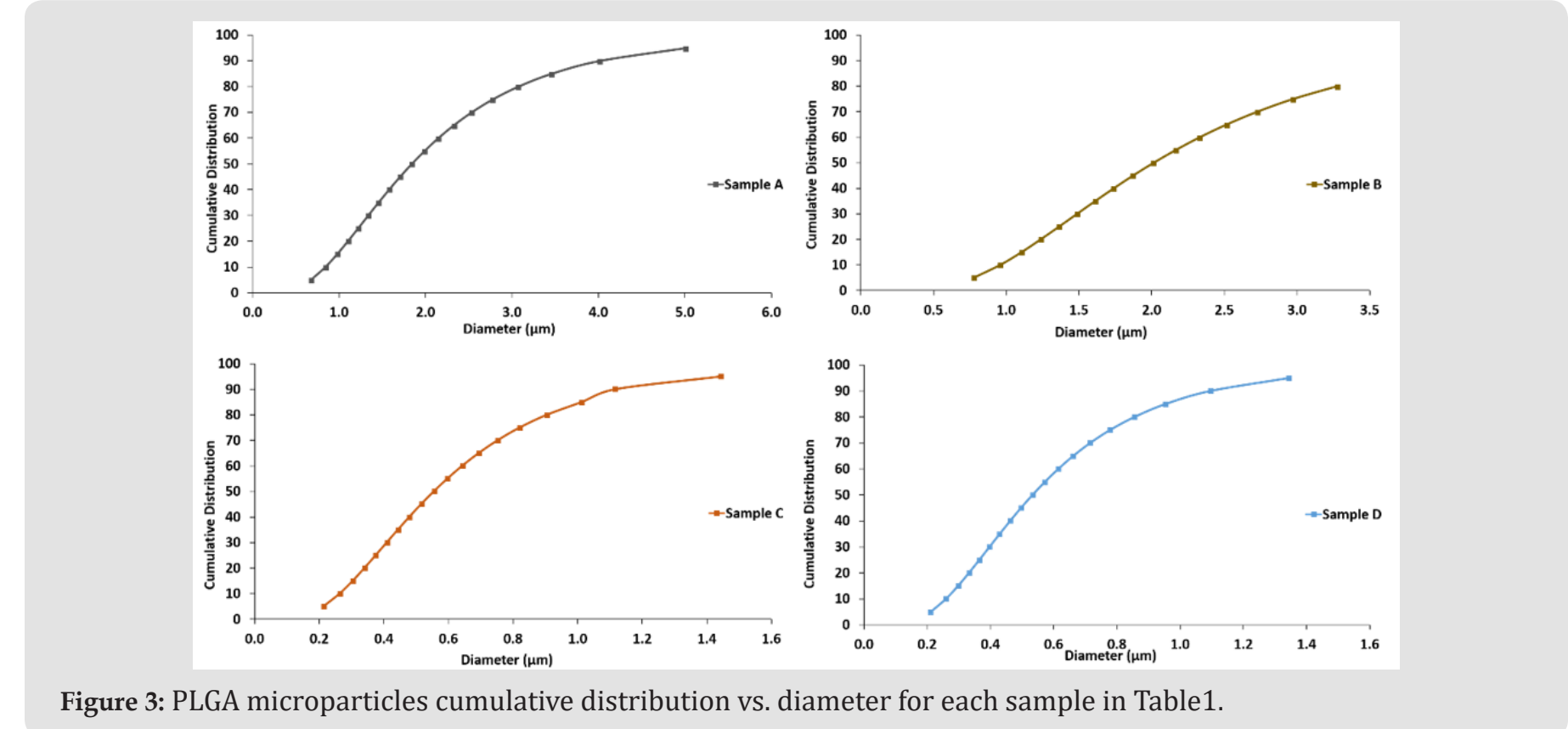

with $0.1 \%, 0.5 \%$ and $3 \% \mathrm{NaHCO}_{3}$ was $0.63 \pm 0.32 \mu \mathrm{m}$ (polydispersity $=0.40$ ) $1.80 \pm 0.66 \mu \mathrm{m}$ (polydispersity $=0.40$ ), and $2.11 \pm 1.15 \mu \mathrm{m}$ (polydispersity $=0.45)$, respectively. The mean diameter of the PLGA microparticles increased with the addition of $\mathrm{NaHCO}_{3}$. Figure 3 shows PLGA microparticle's cumulative distribution vs. diameter of each sample. 


\section{Nanoporous PLGA Microparticle Porosity Analysis}

PLGA microparticles samples porosity has been calculated using ImageJ software (National Institutes of Health, Bethesda, Maryland, USA). Using the analyze particles function within ImageJ, the percentage of pixels in the image have been highlighted by applying the built-in image threshold function (Table 2). Since the pores showed a different threshold than the background, porosity can be calculated in a very efficient technique (Figure 4).

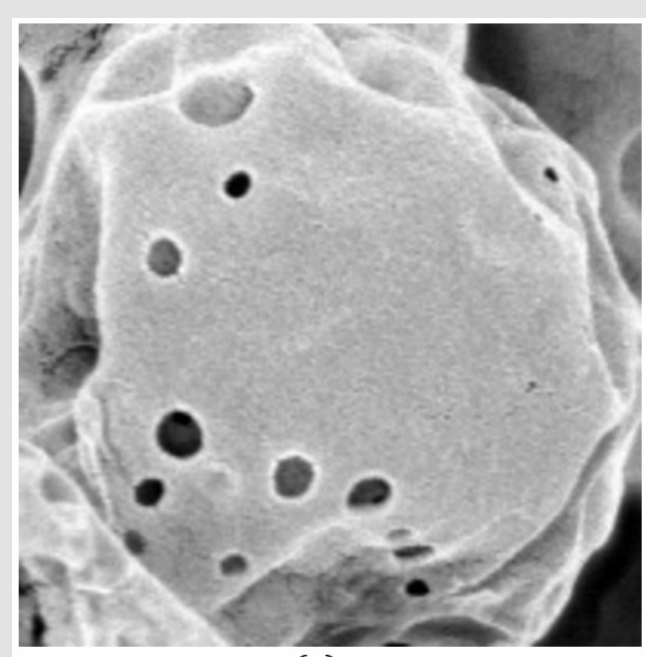

(a)
Table 2: Pore size and porosity (\%) of nano porous PLGA microparticles.

\begin{tabular}{|c|c|c|}
\hline Sample No. & Pores Size (nm) & Porosity (\%) \\
\hline $\mathrm{A}^{1}$ & $103 \pm 56$ & $18.2 \pm 6.1$ \\
\hline $\mathrm{B}^{1}$ & $75 \pm 31$ & $2.1 \pm 2.0$ \\
\hline $\mathrm{C}^{1}$ & $59 \pm 102$ & $0.07 \pm 0.12$ \\
\hline $\mathrm{D}$ & $\mathrm{n} / \mathrm{a}$ & $\mathrm{n} / \mathrm{a}$ \\
\hline
\end{tabular}

${ }^{1}(\mathrm{n}=3)$

Figure 4: Example of the porosity analysis using built-in threshold function in ImageJ. (a) Before applying the threshold function. (b) After applying the threshold function.

\section{Lysine Assay}

The micro BCA assay kit has been used for colorimetric detection and quantitation of total Lysine molecules have attached to nanoporous PLGA microparticles surfaces. The absorbance of the sample at $562 \mathrm{~nm}$ was measured on a plate reader $(\mathrm{n}=3)$, with a control sample of Lysine in PBS only (Figure 5). The data

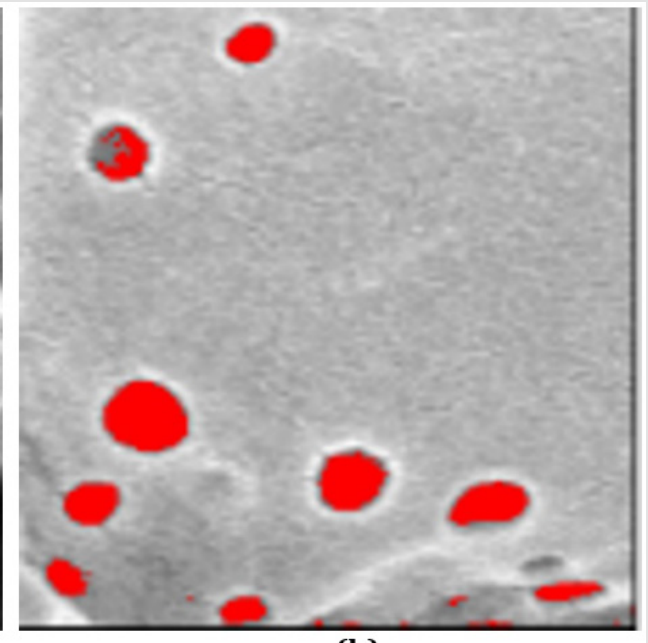

(b) has been collected after $30 \mathrm{~min}$ and after one hour from Lysine addition to nanoporous PLGA microparticles. The assay results showed a correlation between the amounts of Lysine found in each sample with salt concentration used to prepare the sample. A linear response between the salt concentration and the Lysine binding to nanoporous PLGA microparticles surface with a reported $R^{2}=0.94$ (Figure 6).

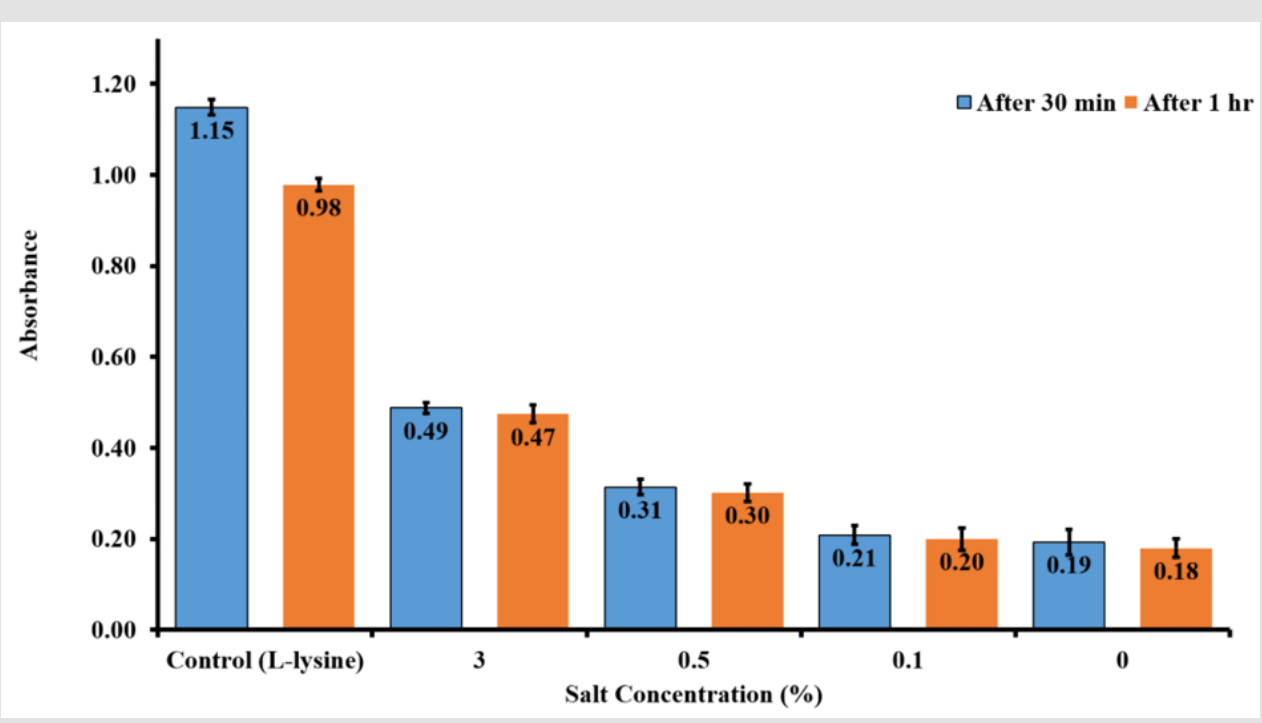

Figure 5: Lysine assay using BCA assay kit for $30 \mathrm{~min}$ and one hour from sample addition. 


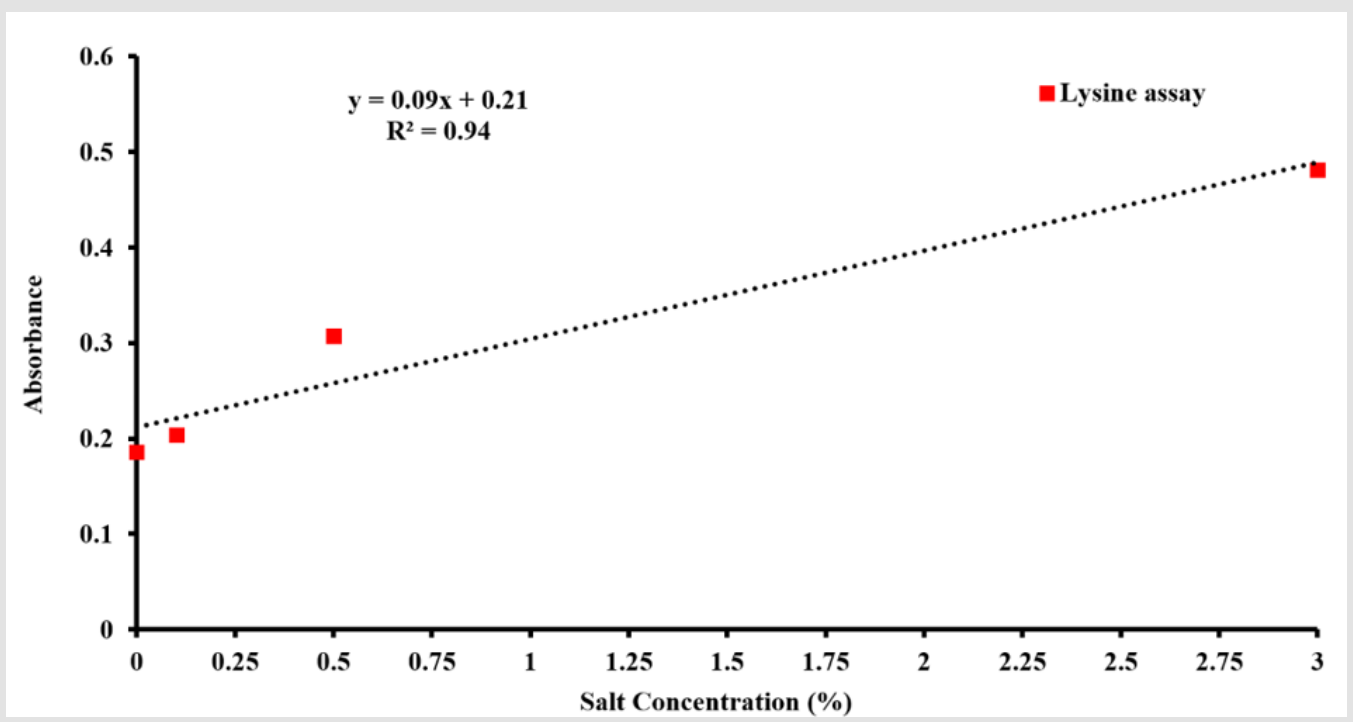

Figure 6: Linear response of Lysine with salt concentration.

\section{Discussion}

The spherical morphology of the synthesized nanoporous PLGA microparticle makes the movement more natural due to their symmetry; therefore, they travel through indirect pathways with fewer collisions [21]. No direct measurement has been conducted to calculate the percentage of PLGA microparticles that became nanoporous because the PLGA microparticles suspended in the solution undergo Brownian motion. Therefore, the motion of the particles is entirely random depends on the size, viscosity, and temperature. Hence, an estimation of the percentage of PLGA microparticles became nanoporous by reporting the cumulative distribution percentage from Figure 3 that reflects mean diameters from each sample. The result shows $\sim 50 \%, 39.8 \%$, and $50.4 \%$ of Sample A, B, and C, respectively, are nanoporous microparticles.

Controlling the nanoporous PLGA microparticles diameter size in such an approach can be very useful for the microparticles applications. For example, platelets (size $2-3 \mu \mathrm{m}$ ) are known to promote metastasis by protecting cancer cells against natural killer cells in the blood, because of direct contact between platelets and targeted cells [22]. The PLGA microparticles size is very close to platelets; this can be used to assist the physical attachment to the targeted cell surface.

Table 2 shows the pore size measurement and porosity percentage calculation for the samples prepared in Table 1 The analysis shows an increase in porosity (\%) as $\mathrm{NaHCO} 3$ concentration increases. Based on the porosity analysis, the process efficiency to produce nanoporous depends on the salt concentration. On the other hand, the pore size is random in this case and does not depend on the salt's concentration. One-way ANOVA test shows a p-value of 0.806 , which means no significant difference between the pore sizes from different samples.

The selective targeting approaches require ligands that specifically interact with receptors expressed on the cell surface. Therefore, this means multiple copies of the ligand on the PLGA microparticles surface to increase the binding effects, which results in higher affinity. The sample with higher porosity showed higher Lysine concentration, which can be translated into more primary amines are attached to the surface. The results show an approach capable of controlling the density of ligand that could conjugate on the microparticle surface to enhance the binding, which results in higher affinity. Also, the results showed a stable covalent attachment of Lysine to nanoporous PLGA microparticles.

\section{Conclusion}

This work demonstrates the method to control the porosity of nanoporous PLGA microparticles. The nanoporous PLGA microparticles surface was activated with BS3 to form a stable amide bond with primary amine groups. The double emulation technique has been used to synthesize the particles. The BS3 crosslinker chemistry has been used to functionalize the nanoporous PLGA microparticles surface. This work shows stable amide bond formation between the BS3 and L-lysine and a novel method to control the amount of Lysine attachment to the surface by controlling the porosity of PLGA microparticles. The nanoporous PLGA microparticles platform can be designed to carry out multifunctional purposes.

Due to the surface area increase, the functionalization of microparticles can maximize receptor selectively targeting, which can be recognized as an advanced therapy for cancer. A higher density of ion channel blockers or any other ligand would be available for specific binding and reduce non-specific binding. The PLGA microparticles have been used to load and deliver drug molecules. The future direction of this platform is to complete studies with ion channel blockers to develop the cell-targeted delivery platform.

\section{Acknowledgment}

The authors acknowledge experimental assistance and useful discussion with Dr. M. R. Hassan, Dr. Sai S. Sasank Peri, Dr. Nuzhat Mansur, and Dr. M. U. Raza. 


\section{Conflicts of Interest}

The authors declare no conflict of interest.

\section{References}

1. Zheng W (2009) A water-in-oil-in-oil-in-water (W/O/O/W) method for producing drug-releasing, double walled microspheres. Int J Pharm 374(1-2): 90-95.

2. Barrow WW (2004) Microsphere technology for chemotherapy of mycobacterial infections. Curr Pharm Des 10(26): 3275-3284.

3. Kapoor DN, Bhatia A, Kaur R, Sharma R, Kaur G, et al. (2015) PLGA: a unique polymer for drug delivery. Ther Deliv 6(1): 41-58.

4. Shive MS, Anderson JM (1997) Biodegradation and biocompatibility of PLA and PLGA microspheres. AdvDrug Deliv Rev 28(1): 5-24.

5. Fredenberg S Wahlgren M, Reslow M, Axelsson A (2011) The mechanisms of drug release in poly lacticco-glycolic acid)-based drug delivery systems---a review. Int J Pharm 415 (1-2): 34-52.

6. Cegnar M, Kristl J, Kos J (2005) Nanoscale polymer carriers to deliver chemotherapeutic agents to tumours. Expert Opin Biol Ther 5(12): 1557-1569.

7. Juillerat-Jeanneret L, Schmitt F (2007) Chemical modification of therapeutic drugs or drug vector systems to achieve targeted therapy: looking for the grail. Med Res Rev 27 (4): 574-590.

8. Halasz I, Horvath C (1964) Porous Layer Glass Bead Column Packing in Gas Adsorption Layer Chromatography. Anal Chem 36(12): 2226.

9. Halasz I, Horvath C (1964) Porous Layer Glass Bead Column Packing in Gas Adsorption Layer Chromatography. Anal Chem 36(7): 1178-1186.

10. Kirkland JJ (1976) Porous Silica Microspheres for High-Performance Size Exclusion Chromatography. J Chromatogr 125(1): 231-250.

11. LaVan DA, Mc Guire T, Langer R (2003) Small-scale systems for in vivo drug delivery. Nat Biotechnol 21(10): 1184-1191.

12. Mohamed F van der Walle CF (2008) Engineering biodegradable polyester particles with specific drug targeting and drug release properties. J Pharm Sci-Us 97(1): 71-87.

ISSN: $2574-1241$

DOI: $10.26717 / B J S T R .2020 .27 .004465$

Mohammad G Abdallah. Biomed J Sci \& Tech Res

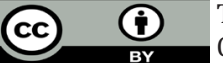

This work is licensed under Creative Commons Attribution 4.0 License

Submission Link: https://biomedres.us/submit-manuscript.php
13. Ilyas A, Islam M, Asghar W, Menon JU, Wadajkar AS, et al. (2013) SaltLeaching Synthesis of Porous PLGA Nanoparticles. Ieee T Nanotechnol 12 (6): 1082-1088.

14. Klose D, Siepmann F, Elkharraz K, Krenzlin S, Siepmann J (2006) How porosity and size affect the drug release mechanisms from PLIGA-based microparticles. Int J Pharmaceut 314(2): 198-206.

15. Hutmacher DW (2000) Scaffolds in tissue engineering bone and cartilage. Biomaterials 21(24): 2529-2543.

16. Chung HJ, Park TG (2007) Surface engineered and drug releasing prefabricated scaffolds for tissue engineering. Adv Drug Deliver Rev 59(4-5): 249-262.

17. Abdallah MG, Yousufuddin M, Yaman S, Khan R, Kim Y, et al. (2017) Surface functionalization of nanoporous PLGA microparticles. 2017 IEEE 12th Nanotechnology Materials and Devices Conference (NMDC): 204-205

18. Lee SB, Kim YH, Chong MS, Hong SH, Lee YM (2005) Study of gelatincontaining artificial skin V: fabrication of gelatin scaffolds using a saltleaching method. Biomaterials 26(14): 1961-1968.

19. Kim TG, Chung HJ, Park TG (2008) Macroporous and nanofibrous hyaluronic acid/collagen hybrid scaffold fabricated by concurrent electrospinning and deposition/leaching of salt particles. Acta Biomater 4(6): 1611-1619.

20. Karp F, Busatto C, Turino L, Luna J, Estenoz D (2019) PLGA nano- and microparticles for the controlled release of florfenicol: Experimental and theoretical study. J Appl Polym Sci 136: (12).

21. Almeria B, Deng WW, Fahmy TM, Gomez A (2010) Controlling the morphology of electrospray generated PLGA microparticles for drug delivery. J Colloid Interf Sci 343 (1): 125-133.

22. Kocbek P, Obermajer N, Cegnar M, Kos J, Kristl J (2007) Targeting cancer cells using PLGA nanoparticlessurface modified with monoclonal antibody. J Control Release 120(1-2): 18-26.

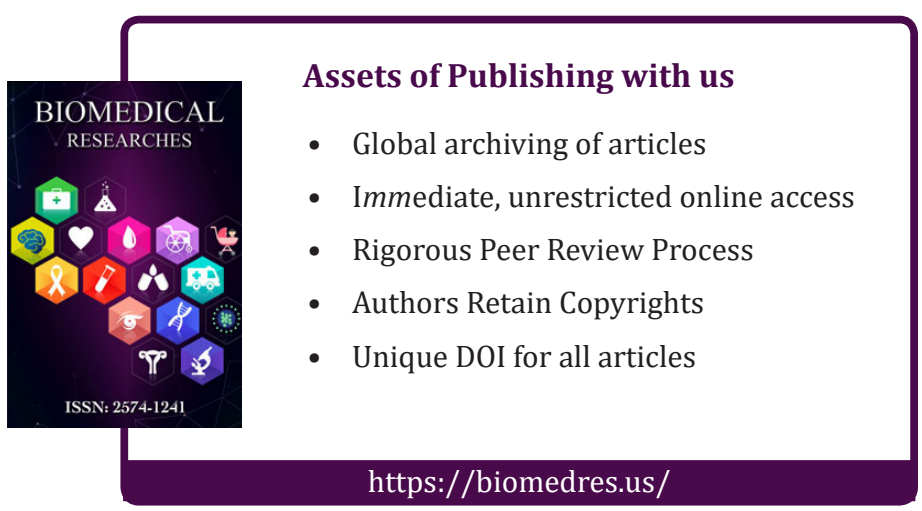

